\title{
Self-supervised Clustering of Mass Spectrometry Imaging Data Using Contrastive Learning
}

Hang $\mathrm{Hu}^{1}$, Jyothsna Padmakumar Bindu ${ }^{2}$ and Julia Laskin ${ }^{1 *}$

1. Department of Chemistry, Purdue University, West Lafayette, IN 47907, USA

2. School of Engineering Technology, Purdue University, West Lafayette, IN 47907, USA

Corresponding author: Julia Laskin, Tel: 765-494-5464, Email: jlaskin@purdue.edu

\begin{abstract}
Mass spectrometry imaging (MSI) is widely used for the label-free molecular mapping of biological samples. The identification of co-localized molecules in MSI data is crucial to the understanding of biochemical pathways. However, complex MSI data are too large for manual annotation but too small for training deep networks. Herein, we introduce a self-supervised clustering approach based on contrastive learning, which shows an excellent performance in clustering of small MSI data. We train a deep convolutional neural network $(\mathrm{CNN})$ using MSI data from a single experiment without manual annotations to effectively learn high-level spatial features from ion images and classify them based on molecular colocalizations. We demonstrate that contrastive learning generates ion image representations that form well-resolved clusters. Subsequent self-labeling is used to fine-tune both the CNN encoder and linear classifier based on confidently classified ion images. This new approach enables autonomous and highthroughput identification of co-localized species in MSI data, which will dramatically expand the application of spatial lipidomics, metabolomics, and proteomics in biological research.
\end{abstract}

\section{Introduction}

Mass spectrometry imaging (MSI) is a powerful label-free molecular imaging technique for biological research, which enables simultaneous localization of multiple classes of biomolecules with high sensitivity and unprecedented molecular specificity. ${ }^{1-4}$ By acquiring a full mass spectrum in each pixel of a virtual grid, MSI generates hundreds of molecular images in a single experiment. Recent advances in MSI technology focus on the enhancement of the spatial resolution, ${ }^{5,6}$ depth of molecular coverage ${ }^{7-9}$ and acquisition throughput, ${ }^{10-12}$ all of which substantially increase the data size. The interpretation of complex MSI data is a major bottleneck on the path to scientific discovery, which motivates the development of computational tools for data mining and visualization ${ }^{13,14}$.

A recurring task in MSI data analysis is to identify co-localized molecules, which is critical to the identification of key biochemical pathways of interest to biomarker discovery, ${ }^{15,16}$ drug development,,${ }^{17,18}$ and clinical diagnostics. ${ }^{19-21}$ Previous computational approaches used vector-based similarity measurements to determine molecular colocalizations. ${ }^{22-25}$ However, these methods cannot correlate highlevel spatial features making them disproportionately sensitive to the experimental artifacts and noise, which reduces their generalization capacity towards spatial patterns with similar localization but different contrast. Recently, transfer learning and semi-supervised deep learning approaches using convolutional neural network (CNN) have been developed to cluster ion images and quantify the molecular colocalization, respectively ${ }^{26,27}$. These reports indicate that the limited size of MSI data presents a challenge to conventional CNN training frameworks, which typically rely on a large number of annotated images. As a result, these approaches provide a relatively minor improvement over the traditional machine learning methods for finding co-localized molecular images. 
Recent advances in self-supervised contrastive learning approaches for computer vision including $\mathrm{MoCo}^{28}$, $\mathrm{SimCLR}^{29}$ and $\mathrm{SwAV}^{30}$ have opened up new opportunities for learning visual representations without manual annotations. In natural image classification, these approaches provide comparable results to those obtained using supervised learning. Herein, we demonstrate that contrastive learning may be used to overcome the existing gap in the classification of MSI data due to the limited data size. We introduce a robust self-supervised clustering approach, which enables efficient colocalization of molecules in individual MSI data by retraining a CNN and learning representations of high-level molecular features in these data.

\section{Results and discussion}

\section{Self-supervised training of a CNN model for molecular localization clustering}

The self-supervised approach for molecular colocalization developed in this study is illustrated in Fig. 1. The approach is based on training a CNN to learn representations of molecular localizations and classify molecular images into groups based on high-level spatial features. The clustering results provide a concise presentation of the spatial patterns present in large MSI data, which is critical to understanding the relevant biochemical pathways. To facilitate the autonomous and high-throughput MSI-based scientific discovery, we train our model in a self-supervised manner without manual annotations. This is achieved using image augmentation, which enables an effective self-supervised training of a deep CNN with a limited number of ion images. The self-supervised clustering approach developed in this study is summarized in Fig. 1. The approach relies on the following three steps described in detail later: 1) Contrastive learning of molecular localization representations using SimCLR; 2) Image clustering based on the representations and 3) Selflabeling of the clustered images.

1. Contrastive learning

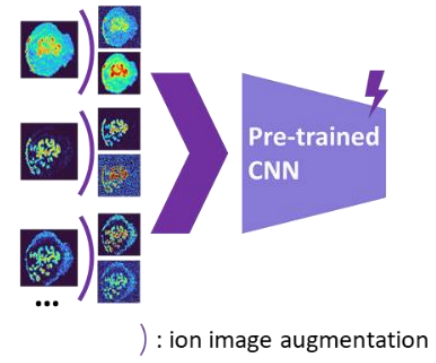

2. Image clustering

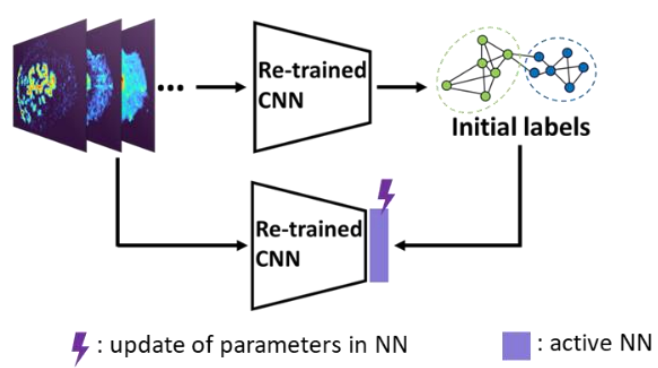

3. Self-labeling

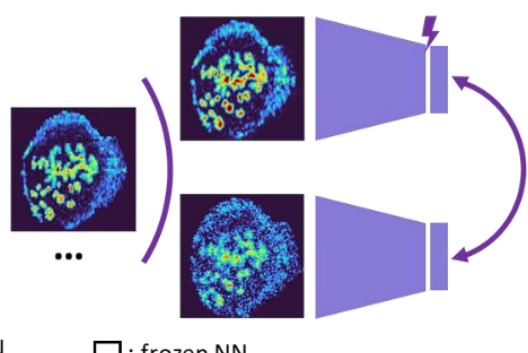

Figure 1. self-supervised training of CNN model for molecular colocalization. (1) CNN encoder is trained by contrastive learning to learn ion image representations. (2) Learned image representations are classified by spectral clustering. This classification pretext task is utilized to initiate a linear classifier after CNN encoder. (3) The whole CNN model is further fine-tuned using self-labeling.

In order to assess the improvement of the model during the self-supervised training, we systematically evaluated each training step using a manually annotated benchmark MSI dataset of a mouse uterine tissue acquired using nanospray desorption electrospray ionization (nano-DESI). ${ }^{31}$ The mouse uterine tissue with several distinct cell types is an excellent model system, which presents diverse molecular localizations. From the data acquired in both positive and negative ionization modes, we manually selected 367 ion images (96 x 96 pixels) and clustered them into 13 classes (Methods). We then validated our approach using a publicly available mouse brain tissue MSI dataset from METASPACE ${ }^{32}$. It is acquired using matrixassisted laser desorption/ionization (MALDI), which contains 1101 high resolution ion images (224 x 224 pixels) without annotations. Our results demonstrate the robustness of the self-supervised image clustering approach for MSI datasets of different sizes, spatial resolutions, tissue types, and acquisition conditions. 


\section{Contrastive learning of image representations}

In the contrastive learning step, we use SimCLR to train a CNN encoder for learning image representations. We used EfficientNet-B0 trained on ImageNet as a baseline CNN. EfficientNet ${ }^{33}$ has been demonstrated to achieve high accuracy on ImageNet and provide an order of magnitude higher efficiency than previous models, such as ResNet and Xception. In SimCLR framework (see ESI, Fig. S1), a mini-batch of N ion images is sampled and each image is subjected to a pair of stochastic transformations to generate $2 \mathrm{~N}$ augmented images. A positive augmented pair is derived from the same ion image. Meanwhile, the remaining $2(\mathrm{~N}-1)$ augmented images are treated as negative instances. SimCLR learns visual representations by maximizing the similarity between the positive pair of images while minimizing their similarity to the negative instances via a contrastive loss in the latent space. Details of the framework are described in the Methods section.

Data augmentation plays a critical role in the training step. It ensures that the learned visual representations of ion images are independent of the employed transformations. This generalization power of SimCLR is critical to learning high-level spatial features instead of pixel-level details. In order to evaluate the performance of this step, we systematically investigated the impact of image augmentation operations on image classification in the benchmark dataset as shown in Fig. 2a. In particular, we used Gaussian blur, Gaussian noise, and intensity distortion to alter the appearance of ion images along with translation, random resized crop, and rotation to alter their geometry. For each type of augmentation, we performed SimCLR using the same training protocol and evaluated the learned representations using a linear evaluation, in which the accuracy describes the quality of the representation (Methods). We also examined the performance of SimCLR and transfer learning in the absence of augmentation for comparison. Fig. 2b shows that all the appearance-changing augmentations improve the performance of the representation learning. Meanwhile, all the geometry-changing augmentations except for rotation do not provide a measurable improvement. Stronger geometric transformations reduce the classification accuracy (Fig. S2). This observation indicates that in contrast to the semantic classification of natural images, strong alteration of the geometry of ion images is detrimental to representation learning of molecular localizations. We also examined the combined effect of the appearance-changing augmentations on the learned representations. Fig. $2 b$ shows that a combination of three appearance-changing augmentation operators results in $>80 \%$ accuracy in the linear evaluation. An example shown in Fig. S3 illustrates the power of the generalization provided by this augmentation strategy. In particular, for ion images that have different contrast and noise level, augmented images generated for one molecule $(\mathrm{m} / \mathrm{z} 789.561$ in positive mode) become similar to the original images of other molecules ( $\mathrm{m} / \mathrm{z} 746.5108$ in positive and 599.3205 in negative modes, respectively). As a result, these molecules are classified into one group in the self-supervised clustering process. Our results indicate that, for MSI data, the generalization power of contrastive learning stems from the appearance-changing image augmentations. 
a

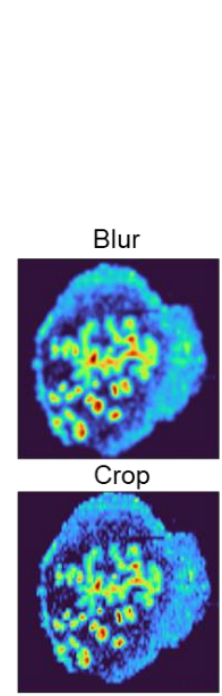

C

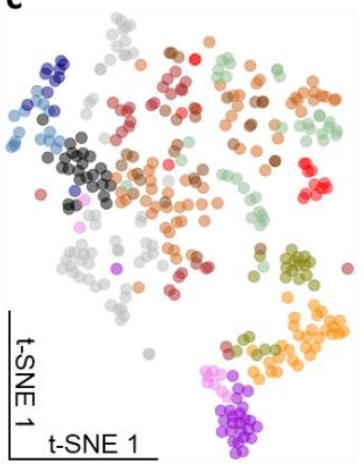

f

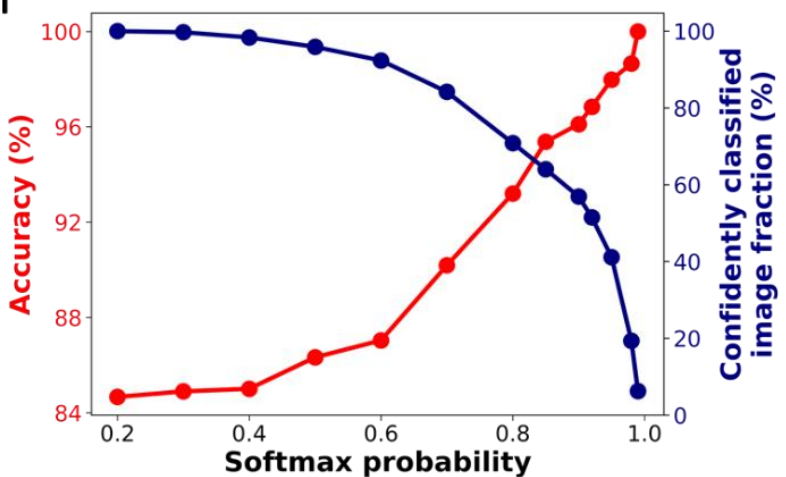

d b

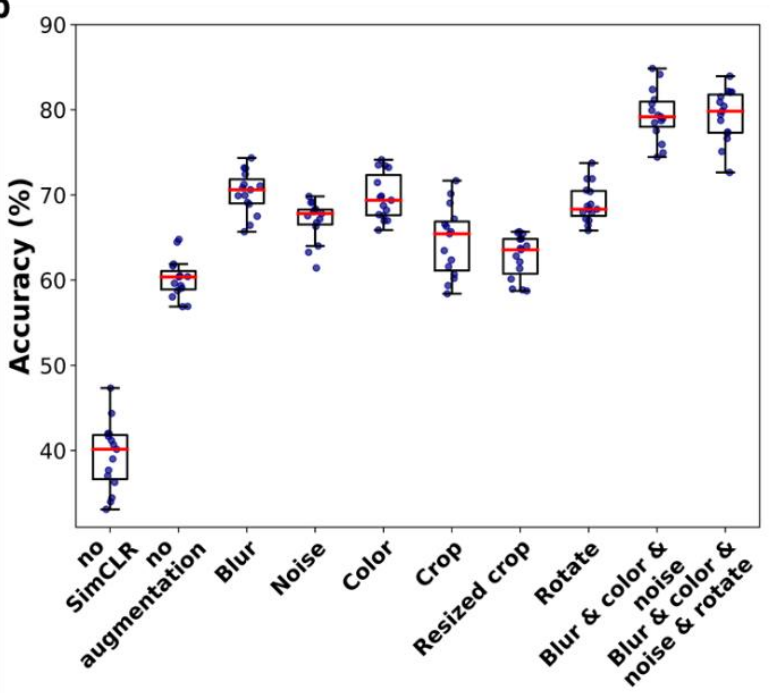

e

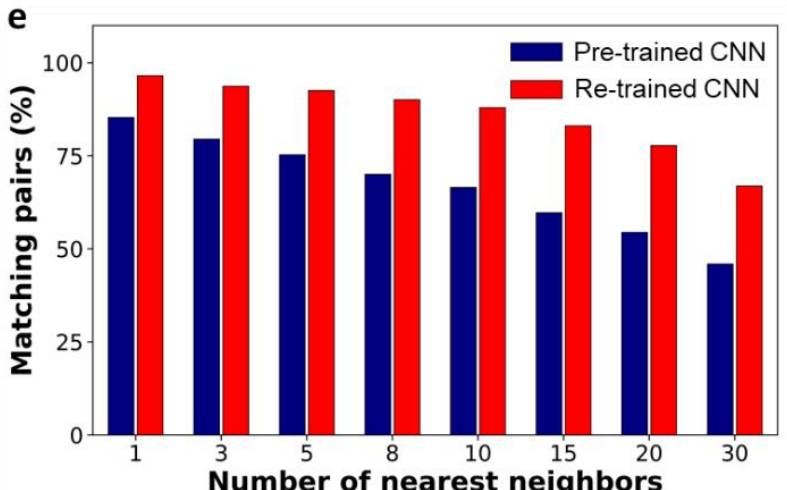

g

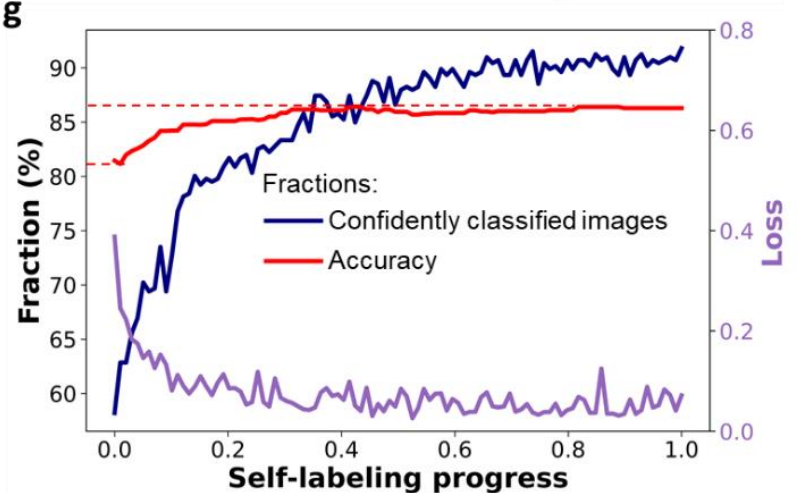

Figure 2. Self-supervised clustering enables effective molecular localization representation learning and classification in benchmark data. (a) Illustration of studied image augmentation operators. (b) Linear evaluation of re-trained CNN encoder with individual or composite image augmentation operators. t-SNE visualizations of ion image representations obtained from (c) pre-trained CNN encoder and (d) re-trained $\mathrm{CNN}$ encoder. Each data point corresponds to an ion image. (e) Contrastive learning substantially improves the purity of local neighborhoods of ion images in the representation space. (f) The relationship between classification accuracy and fraction of confidently classified ion images, which are selected based on a series of softmax probability thresholds. (g) Changes in the training loss, accuracy, and number of confidently classified ion images during the self-labeling process. 
The learned representations for the benchmark dataset are visualized using t-SNE in Figs. $2 \mathrm{c}$ and $2 \mathrm{~d}$ with the color coding obtained from the manual image classification. The results demonstrate that the pre-trained EfficientNet-B0 model does not separate different classes of ion images (Fig. 2c). In contrast, the separation and compactness of clusters are dramatically improved using the re-trained encoder (Fig. 2d). These findings indicate that contrastive learning provides meaningful localization representations, which may be used for image clustering without annotations. We also studied the impact of training time on the learned representations as shown in Fig. S4. Because the algorithm maximizes the similarity of positive pairs and minimizes the similarity of negative instances, we observe a trade-off between the alignment and uniformity in the learned image representations ${ }^{34}$. Alignment indicates that feature vectors of two images from a positive pair should be mapped together while uniformity indicates that all feature vectors should be uniformly distributed. For the benchmark dataset, alignment dominates the training process in the first 50 epochs, in which ion images from the same class tightly aggregate together in the 2D feature space (Fig. S4a). Further training beyond this point disproportionately increases the uniformity of data distribution, which is detrimental to the downstream classification. In addition, a fast decrease in the contrastive loss observed in the first 50 epochs is followed by a much slower trend at longer training times (Fig. S4b) indicating the diminished benefit of a longer training. The linear evaluation results shown in Fig. S4c indicate that 50 epochs of training provide the best classification of the benchmark data.

\section{Image clustering}

In the second step illustrated in Fig. 1, we performed image clustering based on the representations and generated the initial classification labels for the self-labeling task. Spectral clustering (SC) approach is selected, which constructs a k-nearest neighbor graph from ion image representations and then identifies clusters through the Laplacian embedding. Because contrastive learning provides image representations with meaningful local neighborhoods, SC is an appropriate method for this task ${ }^{35}$. For representations of benchmark dataset given by contrastive learning, we quantified the purity of local neighborhoods by counting the annotation-matching pairs for each image and its k-nearest neighbors, where k ranges from 1 to 30. (Methods) Our results confirm that contrastive learning substantially improves the purity of local neighborhoods of ion images in the representation space as shown in Fig. 2e. In particular, we observe that for a relatively large neighborhood size $(\mathrm{k}>3)$, the re-trained encoder improves the pair-matching percentage by more than $15 \%$. For example, for ten nearest neighbors, the pair-matching percentage is $88 \%$ and $66 \%$ for the re-trained and pre-trained encoders, respectively. A combination of contrastive learning and SC provides $81.5 \%$ classification accuracy for benchmark dataset with 13 clusters as shown in Table 1. However, this machine learning classifier is non-learnable, which hinders further model improvement. In order to further enhance the clustering performance, we used the initial labels obtained from SC to initialize a learnable linear classifier at the end of the CNN encoder and then fine-tuned the whole model using a self-labeling approach ${ }^{36}$. This classifier is composed of a linear layer followed by a softmax function. Its initialization is performed by training it on top of the frozen encoder with the original ion images and initial labels as inputs as illustrated in step 2 of Fig. 1.

\section{Self-labeling}

The self-labeling step shown in Fig. 1 fine-tunes both the CNN encoder and linear classifier by ensuring that augmentations of the same ion image are be classified into the same group. This approach further enhances the generalization power of the model, which becomes tolerant towards visual variations originating from strong data augmentations (Methods). To optimize the training process, only confidently classified images are included in self-labeling. 
Because the initial labels are generated using an unsupervised machine learning approach, we anticipate that some false classification may be present. We identified falsely classified images based on their softmax probabilities $^{37}$. By excluding these images from training, we improved the robustness of the CNN model, which benefits the classification accuracy. In order to select images with correct classification during the training, we first examined the relationship between the softmax probability and classification accuracy for the CNN model using the benchmark dataset. This model was trained by initial labels obtained from SC and classified ion images into 13 classes. We used a range of softmax probability thresholds to examine the classification accuracy (red trace) and fraction of confidently identified images (blue trace) as shown in Fig. $2 \mathrm{f}$. We observe that the classification accuracy increases with increase in the softmax probability threshold. Meanwhile, the number of confidently classified images decreases. Additional examples of this analysis are shown in Fig. S5 indicating that the observed trend is general.

The results shown in Fig. $2 \mathrm{f}$ indicate that there is a trade-off between the number of confidently classified images and classification accuracy. In self-labeling, we chose a probability threshold of 0.9 to start training, for which $58 \%$ of confidently classified images were selected with $96 \%$ classification accuracy. Selflabeling is performed by re-training both the $\mathrm{CNN}$ encoder and classifier using selected images. For each ion image, we use one weak and one strong data augmentation (Tab. S1 and Methods), which provides two pseudo labels as the classifier outputs. A cross-entropy loss is calculated for the pseudo labels and the model parameters are updated to minimize the loss as illustrated in Fig 1. In each epoch, we update the confidently classified images for training using the same softmax probability threshold of 0.9. As illustrated in Fig. 2g, the loss (purple line) decreases with training time. Meanwhile we observe a significant increase in the number of confidently classified images and a slight increase in the accuracy with training time. These results demonstrate that the CNN model corrects itself during the self-labeling process, which gradually includes additional confidently classified ion images into the training and increases the overall classification accuracy.

We used the self-supervised clustering approach to cluster benchmark ion images of the mouse uterine tissue (Fig. S6) into 13 and 20 groups. The results obtained at different stages of the workflow for five replicates are summarized in Table 1. When clustering is performed using the CNN encoder and SC, contrastive learning (SimCLR) improves the classification accuracy from $64.8 \%$ to $81.5 \%$ with 13 clusters and from $71.9 \%$ to $90.0 \%$ with 20 clusters. An improvement of about $20 \%$ in accuracy clearly indicates the significance of the $\mathrm{CNN}$ retraining for learning image representations in MSI data. In addition, self-labeling provides a 3\% improvement in the classification accuracy for both 13 and 20 clusters. Collectively, our self-supervised clustering approach enabled clustering of the benchmark data into 20 groups with 92.7\% accuracy as shown in Fig. S7. Representative ion images for each group shown in Fig. 3 provide a concise summary of the spatial patterns present in the vast MSI data. Meanwhile, the generalization power of the self-supervised clustering approach and its tolerance to noise levels can be assessed by examining images in each class (Fig. S6).

Table 1. Summary of the performance of different clustering methods on benchmark data.

\begin{tabular}{lcc} 
Clustering method & Number of clusters & Accuracy (\%) \\
\hline EfficientNet-B0 + SC & 13 & $64.8 \pm 0.4$ \\
SimCLR + SC & 13 & $81.5 \pm 3.4$ \\
SimCLR + SC + Self-labeling & 13 & $\mathbf{8 4 . 0} \pm \mathbf{3 . 1}$ \\
EfficientNet-B0 + SC & 20 & $71.9 \pm 0.2$ \\
SimCLR + SC & 20 & $90.0 \pm 2.8$ \\
SimCLR + SC + Self-labeling & 20 & $\mathbf{9 2 . 7} \pm \mathbf{2 . 1}$
\end{tabular}




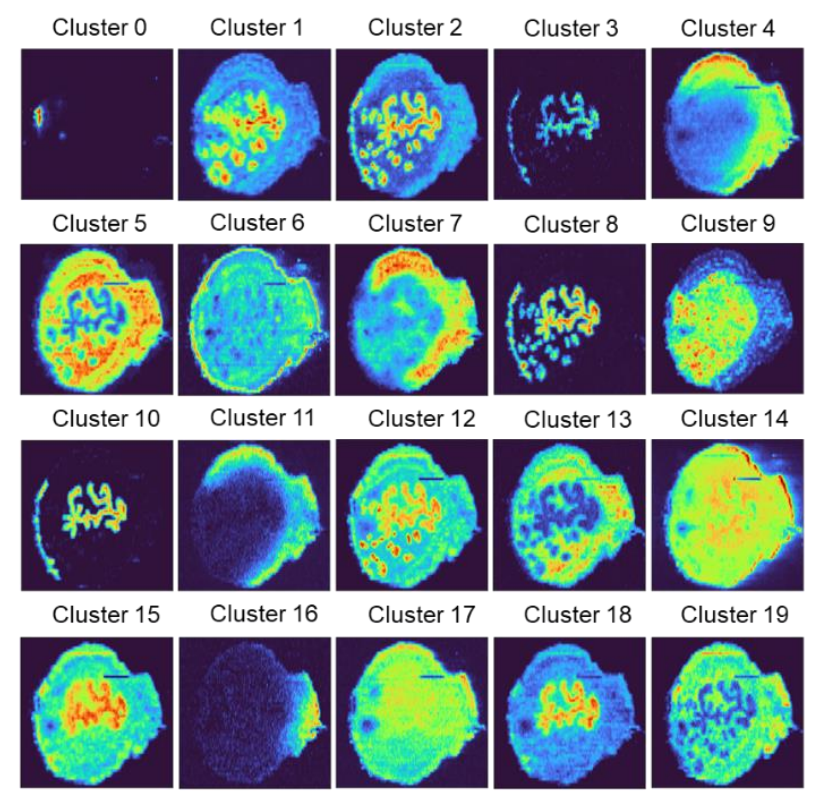

Figure 3. 20 average ion images obtained from self-supervised clustering results provide a concise summary for comprehensive molecular distribution patterns present in the benchmark MSI data.

\section{Mass spectrometry image clustering of an unannotated mouse brain dataset}

To further demonstrate the robustness of the self-supervised clustering approach, we applied it to a publicly available mouse brain MALDI MSI dataset. The image size of 240 × 220 pixels is larger than the benchmark data. For the mouse brain MSI data, we generated 1101 ion images Shown in Fig.S8. We observe diverse spatial patterns of metabolites and lipids localized to different regions of the brain tissue. Ion images showing signal enhancement outside of the tissue region most likely correspond to matrix peaks. Using self-supervised clustering approach, we re-trained the CNN model and clustered 1101 ion images into 35 colocalization groups as shown in Fig. S9. This process took less than one hour with a single GPU card (Methods).

Fig. 4a illustrates ion image representations after self-supervised learning using t-SNE visualization. Additional results are provided in Fig. S10. In the absence of a manual annotation, we use the same color for all the data points in Fig. S10. With the pre-trained EfficientNet-B0, we could only observe several aggregates at the edge of the 2D ion image representations. However, the uniformly distributed representations in the center of the plot cannot be used for identifying the co-localized ion images (Fig. S10a). After the contrastive learning step, the re-trained CNN encoder provides a substantially improved separation of the representations as shown in Fig. S10b. Fig. 4a shows ion image representations after selflabeling, which are color-coded with predicted colocalization labels. Tight clusters indicate co-localized molecular distribution patterns in the MSI data. Pairs of ion images were selected from clusters and placed around the t-SNE plot. Images from one pair have similar spatial features, while different pairs show distinct molecular localizations. These results confirm that the self-supervised clustering approach developed in this study provides accurate molecular localization representations of distinct spatial patterns observed in MSI data. Notably, some of the paired ion images have different noise levels (e.g., m/z 906.4314 vs m/z 915.4561) or different intensity levels (e.g., $\mathrm{m} / \mathrm{z} 613.3477 \mathrm{vs} \mathrm{m} / \mathrm{z} 817.1050$ ). These results indicate that data augmentations used in the training step provide a sufficient generalization capability for the re-trained CNN model to identify high-level molecular localization. The ability to perform self-supervised clustering of the unannotated MSI data distinguishes our approach from previously reported methodologies ${ }^{25-27}$. 

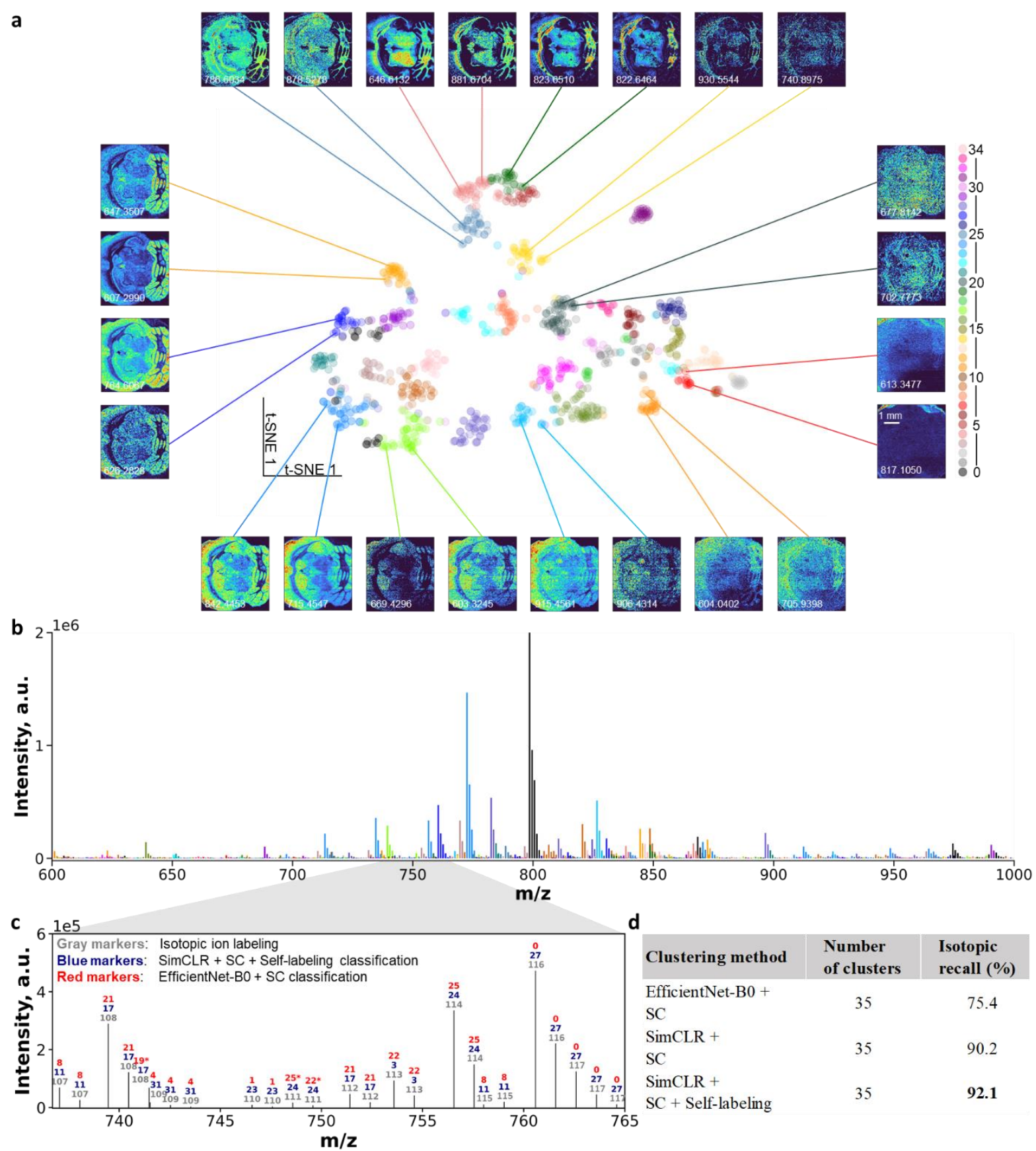

Figure 4. Self-supervised clustering results on a publicly available MALDI mouse brain dataset. (a) $\mathrm{t}-\mathrm{SNE}$ visualization of ion image representations obtained after two steps of self-supervised training. Data points are color-coded using the final clustering assignments of ion images. Pairs of representative ion images are selected from well-resolved clusters to visualize the quality of classification. (b) An average spectrum color-coded using the same color scheme as that in panel a. (c) A zoom in region of the average spectrum showing several isotopic patterns. The results of isotopic identification (ground truth), EfficientNet-B0 and SC classification, and self-supervised clustering classification are annotated using independently assigned class numbers with different colors. Ions with the same color and number are grouped together in the corresponding classification. For clustering results, an asterisk indicates falsely classified isotopic ions. (d) A summary of the isotopic recall for different clustering methods. 
We also visualized the ion clustering results in the $\mathrm{m} / \mathrm{z}$ space. Fig. $4 \mathrm{~b}$ shows an average mass spectrum over the $m / z$ 600-1000 range, in which peaks are highlighted using the same color coding as that in Fig. 4a. To further evaluate the accuracy of the clustering, we examined the isotopic recall ${ }^{26}$, which quantifies the percentage of ion images of isotopic peaks correctly grouped together. We identified isotopic ions based on both the accurate $\mathrm{m} / \mathrm{z}$ shift and Pearson correlations of ion images (Methods). For example, in Fig. 4c, co-localized isotopic peaks observed in the $\mathrm{m} / \mathrm{z}$ range of 736-765 are annotated using compound indices in gray color, which are ranked by their primary isotopic $\mathrm{m} / \mathrm{z}$ values. Ion image colocalization results of selfsupervised clustering and EfficientNet-B 0 followed by SC are also annotated by colocalization class number with blue and red colors, respectively. We note that independent class numbers were assigned to these two classification results. With the expectation that isotopic images should be clustered into the same group, we identified the correctly and falsely classified isotopic ions in clustering results (Methods) and marked false isotopic classification with an asterisk. In the mass range shown in Fig. 4c, EfficientNet-B0 and SC falsely classified 3 isotopic peaks, while the self-supervised clustering approach correctly grouped all the isotopic peaks. This result further confirms the accuracy and robustness of the self-supervised clustering. Values of the isotopic recall obtained using different clustering methods are summarized in Fig. $4 \mathrm{~d}$. For the clustering involving the CNN encoder and SC, contrastive learning (SimCLR) improves the isotopic recall from $75.4 \%$ to $90.2 \%$. With the self-labeling, the final model reaches an isotopic recall of $92.1 \%$, which indicates the superior clustering performance of this approach.

\section{Conclusion}

We developed a robust self-supervised clustering approach for classifying co-localized molecular images obtained using MSI. In this approach, data augmentation is combined with contrastive learning and selflabeling methods to train a deep CNN model without manual annotations. Systematic studies using a fully annotated mouse uterine tissue data and unannotated mouse brain tissue data demonstrate that the re-trained CNN model efficiently learns high-level molecular localization representations, which facilitate clustering of molecular images. Using a manually annotated benchmark dataset, we demonstrate that this approach achieves $>90 \%$ classification accuracy. Meanwhile, clustering of a publicly available unannotated MSI data demonstrates the robustness of this approach and its applicability to different tissue types, image sizes, modes of ionization, instrument parameters, and data complexity.

Our findings indicate that the limited size of MSI data is not a bottleneck for self-supervised learning. However, data augmentation is critical to the model training. We use a combination of appearance-changing transformations, such as Gaussian blur, Gaussian noise and intensity distortion to maximize the generalization power of the CNN model towards the efficient recognition of distinct localization patterns in ion images with varying levels of signal and noise. A similar self-supervised learning paradigm may be applied to other hyperspectral chemical imaging modalities including Raman and infrared microscopy.

The self-supervised learning approach presented herein enables molecular colocalization analysis based on the MSI data in an autonomous and high-throughput manner. It provides a concise representation of the vast data containing several hundreds of molecular images, which is critical to understanding biochemical pathways in biological systems. Furthermore, we propose that this approach may be readily expanded into a larger semi-supervised learning framework. The self-supervised paradigm enables representation learning before supervised classification, which is particularly advantageous for automatic ion image labeling necessary for the high-throughput annotation of both MSI data and data obtained using other imaging modalities.

\section{Methods and Materials}

Mass spectrometry imaging data 
Mouse uterine and brain tissue MSI datasets used as examples in this study have been previously reported ${ }^{5,31}$. Briefly, mouse uterine tissue was analyzed using nano-DESI MSI on a Q-Exactive HF-X Orbitrap mass spectrometer (Thermo Fisher Scientific, Waltham, MA) equipped with a custom-designed nano-DESI source $^{38}$. Mass spectra were acquired in the $\mathrm{m} / \mathrm{z}$ range of 133-2000 in both positive and negative ion modes with a spatial resolution of $10 \mu \mathrm{m}$. Positive mode MSI data for mouse brain tissue was obtained from METASPACE ${ }^{32}$, a community resource that provides open access to MSI data. The specific data were acquired using MALDI MSI in the $\mathrm{m} / \mathrm{z}$ range of $600-1000$ with a spatial resolution of $20 \mu \mathrm{m}$. The dimensions of the two MSI datasets are listed in Table S1.

\section{Data pre-processing}

To generate ion images from MSI data, we used peak detection and $\mathrm{m} / \mathrm{z}$ binning as described in our previous study $^{39}$. The signal intensity for each $\mathrm{m} / z$ in each pixel was extracted from the corresponding mass spectrum with a bin width of $\pm 10 \mathrm{ppm}$ and normalized to the total ion current. To remove visual spikes, pixels with intensities > 0.999 quantile were reassigned with the 0.999 intensity quantile value. Ion images of mouse uterine tissue were resized to 96 x 96 pixels. Meanwhile, larger-size ion images of mouse brain tissue were resized to 224 x 224 pixels. Most pre-trained CNN models and Pytorch transform functions accept RGB images. Thus, raw pixel intensities of ion images were normalized between 0 and 255 and copied to 3 channels. More specifically, we converted ion images into the PIL format before the data augmentation step. To benchmark our approach, we manually selected 367 mouse uterine ion images with distinct ion distributions and clustered them into 13 groups according to molecular colocalizations as shown in Fig. S6. For the unannotated mouse brain dataset, we detected 1101 peaks from the average spectrum and generated the corresponding ion images as shown in Fig. S8.

\section{Architecture of the self-supervised clustering}

We approached the challenge of molecular localization clustering as an image classification task. We aimed to re-train a CNN model for an individual MSI dataset to classify ion images based on the high-level spatial features without manual annotations. The model architecture is shown in Fig. 1. The pre-trained CNN (EfficientNet-B0) is re-trained by contrastive learning and self-labeling sequentially in a self-supervised manner. We use EfficientNet-B0, which has been trained on the ImageNet database. We used the EfficientNet-B0 model before the classification layer as an encoder. In our architecture, we firstly learned ion image representations through the contrastive learning. More specifically, SimCLR ${ }^{29}$ approach is adopted in this study. After this first phase of training, we fed ion images through the re-trained encoder to produce a set of feature vectors, which were then passed to a spectral clustering (SC) classifier to generate the initial labels for the classification task. To initialize self-labeling, a linear classifier (a linear layer followed by a softmax function) was attached to the encoder and trained with the original ion images and initial labels as inputs. Finally, we utilized a self-labeling ${ }^{36}$ approach to fine-tune both the encoder and classifier, which allows the network to correct itself.

\section{Contrastive learning}

Details of SimCLR implementation are shown in Fig. S1. The representation of an ion image is the output before the classification layer of EfficientNet-B0. A small multilayer perceptron with one hidden layer maps the representations to the projection space $(Z)$ where the contrastive loss is applied. In the training step each ion image is used to generate a pair of augmentations. We treat two augmentations of one ion image as a positive pair $(i, j)$. Then the loss function is defined as 


$$
\ell_{i, j}=-\log \frac{\exp \operatorname{sim} \mathbf{z}_{i}, \mathbf{z}_{j} / \tau}{\sum_{k=1}^{2 N} \mathbf{1}_{[k \neq i]} \exp \operatorname{sim} \mathbf{z}_{i}, \mathbf{z}_{k} / \tau}
$$

where $2 \mathrm{~N}$ is the number of augmented images, $\mathbf{z}_{i}, \mathbf{z}_{j} \in Z$, sim denotes the cosine similarity, $\mathbf{1}_{[k \neq i]}$ denotes an indicator function evaluating to 1 iff $k \neq i$ and $\tau$ is a temperature parameter with a default value of 0.5 . The final loss is computed across all positive pairs in the minibatch. To evaluate the quality of learned representations for mouse uterine benchmark, we used a linear evaluation and the nearest neighbor mining. In the linear evaluation, one average ion image was generated from each manually classified group of images as the centroid of the cluster. A linear classifier was subsequently trained on top of the frozen encoder with 13 average ion images and their corresponding annotated labels. Next, all original ion images were classified by the $\mathrm{CNN}$ encoder and the updated linear classifier. The resulting classification accuracy is used as a proxy for the quality of image representation ${ }^{29}$. In the nearest neighbor mining protocol, for each ion image, we searched its $K$ nearest neighbors $(K \in[1,30])$ based on cosine distance in the representation space. We quantified the purity of the neighborhood by counting the annotation-matching pairs for each image and their nearest neighbors.

\section{Image clustering}

It has been demonstrated in a previous report ${ }^{36}$ and this study (Fig. 2e) that images with similar high-level spatial features are mapped together in the representation space using contrastive learning. To leverage the meaningful local neighborhoods, SC was adopted to cluster ion images as the classification pretext task. Based on the cosine distance, 10 nearest neighbors of each ion image were identified to construct a graph. Next, we used the discretization approach to cluster nodes in the graph after the Laplacian embeddings ${ }^{35}$. After the SC, we used the resulting classification labels to initialize a learnable linear classifier on top of the $\mathrm{CNN}$ encoder, which enables the following self-labeling process to fine-tune the model (step 2 in Fig. 1). More specifically, the linear classifier is composed of a linear layer and a softmax function. We set the encoder in frozen mode and trained the classifier with original ion images and initial labels obtained from SC.

\section{Self-labeling}

As reported in a previous study, self-labeling improves the CNN model using a plain criterion: two independently augmented images from one ion should be classified into the same cluster. With this principle, the self-supervised training is able to enhance the generalization power of the model. In addition, only the confidently-classified ion images are included in this training ${ }^{36}$. As shown in Fig. $2 \mathrm{f}$ and Fig. S5, we observed that the classification accuracy obtained for selected mouse uterine ion images increases with an increase in the softmax probability threshold. This indicates that the softmax probability threshold may be used to exclude falsely classified ion images from the training, which enhances the accuracy of the CNN model in the self-labeling step. In the implementation, we empirically selected the probability threshold with respect to the sample population. More specifically, after the initialization of the linear classifier, we selected the $40 \%$ quantile of softmax probabilities as the threshold. The selected training data containing $60 \%$ of the original ion images has a larger fraction of correctly classified images than the original data. Training samples were updated with the same probability threshold at every epoch, thus we gradually included more samples into the training (Fig. 2g). For each selected ion image, we applied a weak and a strong augmentations, respectively. Two pseudo labels were obtained after the encoder and classifier. A weighted cross-entropy loss was then applied to the minibatch of weakly augmented ion images to update the parameters of the CNN model. 


\section{Clustering accuracy evaluation}

In this study, we used over-clustering to effectively capture the intra-class variance. The clustering accuracy was calculated using the following equation:

$$
\operatorname{Accuracy}(\mathbb{C}, \mathbb{T})=\frac{1}{N} \sum_{i} \max _{j}\left|c_{i} \cap t_{j}\right|
$$

Where $\mathbb{C}=c_{1}, c_{2}, \ldots, c_{I}$ is the set of predicted clusters, $\mathbb{T}=t_{1}, t_{2}, \ldots, t_{J}$ is the set of ground truth classes, $N$ is the total number of ion images. In each cluster, the most frequent ground truth class was identified and assigned as a predicted class for the whole cluster. The accuracy was calculated by counting the correctly predicted ion images and dividing by $N$.

\section{Isotopic recall evaluation}

Isotopic recall is a metric reported in a previous study ${ }^{26}$ to evaluate the performance of ion image clustering. Isotopic ion images were identified based on the $\mathrm{m} / \mathrm{z}$ shift and Pearson correlation of the ion image with that of the candidate monoisotopic peak. Specifically, mass spectral features separated from each other by $\mathrm{m} / \mathrm{z} 1.003$ with a tolerance of $m / z 0.01$ were assigned as candidate isotopic peaks. Second, ion images of isotopic peaks should have a Pearson correlation greater than 0.5 . For the self-supervised clustering results, we counted the number of isotopic images that were correctly clustered together and divided it by the total number of identified isotopes. This fraction gives the isotopic recall, which is in the range of 0 to 1 .

\section{Data augmentation}

Data augmentation is crucial for contrastive learning and self-labeling. We systematically studied the impact of data augmentation operators as listed in Tab. S2. For each single augmentation operation, we randomly sampled a parameter interval for an image transformation function. Moreover, we classified the compositions of data augmentation operators into three classes. Medium augmentation was used in SimCLR, while weak and strong augmentations were used in self-labeling.

\section{Model training protocal}

For SimCLR training step, we used Adam optimizer with the initial learning rate of 0.001. A cosine annealing with a period of training epochs was used to decay the learning rate. According to the previous report, SimCLR benefits from larger batch sizes ${ }^{29}$. In our implementation, we used the largest batch size allowed by the GPU memory and trained for 100 iterations. For the self-labeling step, an Adam optimizer with the initial learning rate of 0.0001 and the same cosine annealing schedular were used. We trained the CNN for 300 iterations. We implemented the model training on Google Colaboratory, a cloud computing platform. Using the NVIDIA Tesla P100-16GB GPU, the total training time for mouse uterine MSI benchmark and mouse brain MSI dataset was about 15 and 45 minutes, respectively.

\section{Data availability}

Benchmark data reported in this work will be provided soon at https://github.com/hanghu1024/MSI-selfsupervised-clustering. The mouse brain MSI dataset can be obtained from METASPACE (https://metaspace2020.eu). The dataset title is: Mousebrain_MG08_2017_GruppeF.

\section{Code availability}


The source code for the model training and inference with trained weights will be available soon at https://github.com/hanghu1024/MSI-self-supervised-clustering.

\section{References}

1 J. L. Norris and R. M. Caprioli, Chem. Rev., 2013, 113, 2309-2342.

2 A. R. Buchberger, K. DeLaney, J. Johnson and L. Li, Anal. Chem., 2018, 90, 240-265.

3 D. Unsihuay, D. Mesa Sanchez and J. Laskin, Annu. Rev. Phys. Chem., 2020, 72, 307-329.

4 Y. Hu, Z. Wang, L. Liu, J. Zhu, D. Zhang, M. Xu, Y. Zhang, F. Xu and Y. Chen, Chem. Sci., 2021, 12, 7993-8009.

5 M. Kompauer, S. Heiles and B. Spengler, Nat. Methods, 2016, 14, 90-96.

6 M. Niehaus, J. Soltwisch, M. E. Belov and K. Dreisewerd, Nat. Methods, 2019, 16, 925-931.

7 M. R. L. Paine, B. L. J. Poad, G. B. Eijkel, D. L. Marshall, S. J. Blanksby, R. M. A. Heeren and S. R. Ellis, Angew. Chemie - Int. Ed., 2018, 57, 10530-10534.

8 J. M. Spraggins, K. V. Djambazova, E. S. Rivera, L. G. Migas, E. K. Neumann, A. Fuetterer, J. Suetering, N. Goedecke, A. Ly, R. Van De Plas and R. M. Caprioli, Anal. Chem., 2019, 91, $14552-14560$.

9 P. D. Piehowski, Y. Zhu, L. M. Bramer, K. G. Stratton, R. Zhao, D. J. Orton, R. J. Moore, J. Yuan, H. D. Mitchell, Y. Gao, B. J. M. Webb-Robertson, S. K. Dey, R. T. Kelly and K. E. BurnumJohnson, Nat. Commun., 2020, 11, 1-12.

10 A. Tata, A. Gribble, M. Ventura, M. Ganguly, E. Bluemke, H. J. Ginsberg, D. A. Jaffray, D. R. Ifa, A. Vitkin and A. Zarrine-Afsar, Chem. Sci., 2016, 7, 2162-2169.

11 S. S. Basu, M. S. Regan, E. C. Randall, W. M. Abdelmoula, A. R. Clark, B. Gimenez-Cassina Lopez, D. S. Cornett, A. Haase, S. Santagata and N. Y. R. Agar, npj Precis. Oncol., , DOI:10.1038/s41698-019-0089-y.

12 D. Helminiak and C. Engineering, Electron. Imaging, , DOI:10.2352/issn.24701173.2021.15.coimg-290.

13 N. Verbeeck, R. M. Caprioli and R. Van de Plas, Mass Spectrom. Rev., 2019, 1-47.

14 T. Alexandrov, Annu. Rev. Biomed. Data Sci., 2020, 3, 61-87.

15 M. Sans, K. Gharpure, R. Tibshirani, J. Zhang, L. Liang, J. Liu, J. H. Young, R. L. Dood, A. K. Sood and L. S. Eberlin, Cancer Res., 2017, 77, 2903-2913.

16 G. Schleyer, N. Shahaf, C. Ziv, Y. Dong, R. A. Meoded, E. J. N. Helfrich, D. Schatz, S. Rosenwasser, I. Rogachev, A. Aharoni, J. Piel and A. Vardi, Nat. Microbiol., 2019, 4, 527-538.

17 J. Xue, H. Liu, S. Chen, C. Xiong, L. Zhan, J. Sun and Z. Nie, Sci. Adv., , DOI:10.1126/sciadv.aat9039.

18 E. C. Randall, K. B. Emdal, J. K. Laramy, M. Kim, A. Roos, D. Calligaris, M. S. Regan, S. K. Gupta, A. C. Mladek, B. L. Carlson, A. J. Johnson, F. K. Lu, X. S. Xie, B. A. Joughin, R. J. Reddy, S. Peng, W. M. Abdelmoula, P. R. Jackson, A. Kolluri, K. A. Kellersberger, J. N. Agar, D. A. Lauffenburger, K. R. Swanson, N. L. Tran, W. F. Elmquist, F. M. White, J. N. Sarkaria and N. Y. R. Agar, Nat. Commun., , DOI:10.1038/s41467-018-07334-3.

19 P. Inglese, J. S. McKenzie, A. Mroz, J. Kinross, K. Veselkov, E. Holmes, Z. Takats, J. K. 
Nicholson and R. C. Glen, Chem. Sci., 2017, 8, 3500-3511.

20 K. Margulis, A. S. Chiou, S. Z. Aasi, R. J. Tibshirani, J. Y. Tang and R. N. Zare, Proc. Natl. Acad. Sci. U. S. A., 2018, 115, 6347-6352.

21 B. Paul, K. Kysenius, J. B. Hilton, M. M. W. Jones, R. W. Hutchinson, D. Buchanan, C. Rosty, F. Fryer, A. I. Bush, J. M. Hergt, J. Woodhead, D. P. Bishop, P. Doble, M. M. Hill, P. J. Crouch and D. J. Hare, Chem. Sci., , DOI:10.1039/d1sc02237g.

22 L. A. McDonnell, A. Van Remoortere, R. J. M. Van Zeijl and A. M. Deelder, J. Proteome Res., 2008, 7, 3619-3627.

23 C. Kaddi, R. M. Parry and M. D. Wang, Proc. - 2011 IEEE Int. Conf. Bioinforma. Biomed. BIBM 2011, 2011, 604-607.

24 T. Alexandrov, I. Chernyavsky, M. Becker, F. Von Eggeling and S. Nikolenko, Anal. Chem., 2013, 85, 11189-11195.

25 T. Smets, E. Waelkens and B. De Moor, Anal. Chem., 2020, 92, 5240-5248.

26 W. Zhang, M. Claesen, T. Moerman, M. R. Groseclose, E. Waelkens, B. De Moor and N. Verbeeck, Anal. Bioanal. Chem., 2021, 413, 2803-2819.

27 K. Ovchinnikova, L. Stuart, A. Rakhlin, S. Nikolenko and T. Alexandrov, Bioinformatics, 2020, 36, 3215-3224.

28 K. He, H. Fan, Y. Wu, S. Xie and R. Girshick, , Proc. IEEE Comput. Soc. Conf. Comput. Vis. Pattern Recognit. CVPR 2020, 2020, 9729-9738.

29 T. Chen, S. Kornblith, M. Norouzi and G. Hinton, 36th Int. Conf. Mach. Learn. PMLR, 2020, 119, 1597-1607.

30 M. Caron, I. Misra, J. Mairal, P. Goyal, P. Bojanowski and A. Joulin, Adv. Neural Inf. Process. Syst. NeurIPS 2020, 2020, 9912-9924.

31 R. Yin, K. E. Burnum-johnson and J. Laskin, Nat. Protoc., Nat. Protoc., 2019,14, 3445-3470.

32 A. Palmer, P. Phapale, I. Chernyavsky, R. Lavigne, D. Fay, A. Tarasov, V. Kovalev, J. Fuchser, S. Nikolenko, C. Pineau, M. Becker and T. Alexandrov, Nat. Methods, 2016, 14, 57-60.

33 M. Tan and Q. V. Le, 36th Int. Conf. Mach. Learn. PMLR, 2019, 97, 6105-6114.

34 T. Wang and P. Isola, 37th Int. Conf. Mach. Learn. PMLR, 2020, 2020, 119, 9929-9938.

35 U. Von Luxburg, Stat. Comput., 2007, 17, 395-416.

36 W. Van Gansbeke, S. Vandenhende, S. Georgoulis, M. Proesmans and L. Van Gool, Eur. Conf. Comput. Vis. ECCV 2020, 2020, 268-285.

37 D. Hendrycks and K. Gimpel, 5th Int. Conf. Learn. Represent. ICLR 2017, 2017, 1-12.

38 R. Yin, J. Kyle, K. Burnum-Johnson, K. J. Bloodsworth, L. Sussel, C. Ansong and J. Laskin, Anal. Chem., 2018, 90, 6548-6555.

39 H. Hu, R. Yin, H. M. Brown and J. Laskin, Anal. Chem., 2021, 93, 3477-3485. 